\title{
Glass Transition Temperatures in Polymer Mixtures
}

\author{
Constantinos G. PANAYIOTOU \\ Department of Chemical Engineering, University of Thessaloniki, \\ Thessaloniki, 54006, Greece
}

(Received April 23, 1986)

\begin{abstract}
A fluid-lattice theory of fluid mixtures is combined with the Gibbs-DiMarzio approach of zero configurational entropy at glass transition in order to correlate and predict the composition dependence of glass transition temperature, $T_{\mathrm{g}}$, in one-phase polymer mixtures. Such a combination allows an independent quantitative estimation of the significance to $T_{\mathrm{g}}$ of both equation-of-state terms and chain flexibility terms. The model is tested against experimental data on $T_{\mathrm{g}}$ 's for three polymer mixtures known to be compatible in the complete range of composition. The effect of the various binary parameters on glass transition is discussed along with the effect of chain flexibility change upon mixing. It is shown that chain flexibility terms are predominant in determining $T_{\mathrm{g}}$. Two possible ways of taking into account chain flexibility change are proposed.

KEY WORDS Glass Transition / Polymer Mixtures / Statistical

Thermodynamics / Gibbs-DiMarzio Theory / Fluid Lattice /
\end{abstract}

Prediction and correlation of the composition dependence of glass transition temperature in compatible polymer mixtures is a subject of, both, practical and theoretical interest. From the practical point of view a knowledge of $T_{\mathrm{g}}$ is of considerable importance in connection with the processing conditions and the in-service properties of polymer mixtures. From the theoretical point of view the thermodynamic as well as the kinetic character of liquid-glass transition have to be taken into account in fundamental treatments of the phenomenon. Existing treatments are only approximate ones and such a fundamental treatment still remains a challenge to theoreticians.

The rapid accumulation of information on compatible and semicompatible polymer mixtures, especially in the last decade, justifies efforts towards extensions of traditional treatments of glass transition for pure polymers to polymer mixtures. Polymer solution thermodynamics is of great value in these extensions. For engineering calculations, of course, one may resort to simple empirical or semiempirical equations such as the simple Fox equation ${ }^{1}$ or the Couchman equations. ${ }^{2}$ Although the thermodynamic basis of Couchman's equations has been seriously disputed recently, ${ }^{3}$ they have been proved quite successful in a number of cases. ${ }^{2}$ Deviations from linearity with composition of $T_{\mathrm{g}}$ in polymer mixtures may be negative, zero or positive and it is rather too much to expect from these simple equations to be successfull in all these diverse cases. Nevertheless, molecular theories are more appropriate if we, also, wish to gain some physical insight of the liquid-glass transition phenomenon.

Fluid-Lattice models of polymer solutions $^{4-9}$ is a promissing class of models for their thermodynamic properties. A variety of properties of polymer mixtures have been correlated, so far, with these models such as the basic thermodynamic quantities of mixing, upper and lower critical solution temperatures and gas solubilities at various external conditions. On the other hand, in spite of its 
shortcomings, ${ }^{10,11}$ the Gibbs-DiMarzio theory ${ }^{12,13}$ may be considered as one of the best available thermodynamic treatments of the glass transition today. The links between the fluid-lattice models and the GibbsDiMarzio theory have been recently discussed for the case of pure polymers. ${ }^{14}$

In the present work we combine an improved fluid-lattice model for polymer solutions $^{9}$ with Flory's approximate rotational isomeric state model for chain conformations $^{15}$ and with the Gibbs-DiMarzio approach $^{12,13}$ of zero configurational entropy at glass transition in order to estimate the composition dependence of $T_{\mathrm{g}}$ in one-phase polymer mixtures. The fluid-lattice model is used for a separate estimation of the equation-of-state terms from independent information on other thermodynamic properties of the polymer mixtures.

\section{THEORY}

Consider a mixture of $N_{1}$ molecules of type 1 each consisting of $r_{1}$ segments and $N_{2}$ molecules of type 2 each consisting of $r_{2}$ segments at temperature $T$, external pressure $P$, and total volume $V$. Both types of molecules are considered semiflexible. In order to formulate the partition function of the system, we consider that the molecules are arranged on a quasi-lattice of $N_{\mathrm{r}}$ sites, $N_{\mathrm{o}}$ of which are empty. In other words, we consider that the void volume between the chain molecules is evenly divided in $N_{\mathrm{o}}$ holes.

Following Flory, ${ }^{15}$ Gibbs and DiMarzio $^{12,13,16}$ and our previous work ${ }^{9}$ we may write for the system configurational partition function in the $T, P$ ensemble and in its maximum term approximation

$$
\begin{aligned}
Q_{\mathrm{N}}= & \delta_{1}^{N_{1}} \delta_{2}^{N_{2}}\left(\frac{N_{\mathrm{r}}}{N_{\mathrm{o}}}\right)^{N_{\mathrm{o}}}\left(\frac{N_{\mathrm{r}}}{\omega_{1} N_{1} r_{1}}\right)^{N_{1}}\left(\frac{N_{\mathrm{r}}}{\omega_{2} N_{2} r_{2}}\right)^{N_{2}} \\
& \times \exp \left[\frac{-\left(E_{\mathrm{o}}+P V\right)}{R T}\right]
\end{aligned}
$$

where

$$
\omega_{\mathrm{i}}=\frac{\sigma_{\mathrm{i}}}{r_{\mathrm{i}}} \exp \left(r_{\mathrm{i}}-1\right)
$$

and $^{15}$

$$
\begin{aligned}
\delta_{\mathrm{i}}^{N_{\mathrm{i}}}= & \frac{Z_{\mathrm{i}}^{N_{\mathrm{i}}}\left(Z_{\mathrm{i}}-2\right)^{f_{\mathrm{i}} N_{\mathrm{i}}\left(r_{\mathrm{i}}-2\right)}\left[\left(r_{\mathrm{i}}-2\right) N_{\mathrm{i}}\right] !}{\left[f_{\mathrm{i}} \dot{N}_{\mathrm{i}}\left(r_{\mathrm{i}}-2\right)\right] !\left[\left(1-f_{\mathrm{i}}\right) N_{\mathrm{i}}\left(r_{\mathrm{i}}-2\right)\right] !} \\
& \times \exp \left[\frac{-f_{\mathrm{i}} N_{\mathrm{i}}\left(r_{\mathrm{i}}-2\right) \varepsilon_{\mathrm{i}}}{R T}\right]
\end{aligned}
$$

$-E_{\mathrm{o}}$ is the lattice (intermolecular potential) energy of the system, $Z_{\mathrm{i}}$ is the bond (lattice) coordination number (bond conformations) for bond of type i; For simplicity we set $Z_{1}=$ $Z_{2}=Z . \sigma_{\mathrm{i}}$ is a symmetry number and $\varepsilon_{\mathrm{i}}$ is the flex energy for bonds of type $i$, that is the potential energy of the $Z-1$ bent conformations over the favoured conformation. The equilibrium fraction $f_{\mathrm{i}}$ of bonds $\mathrm{i}$ in bent conformations is given by ${ }^{15}$

$$
f_{\mathrm{i}}=\frac{(Z-2) \exp \left(-\frac{\varepsilon_{\mathrm{i}}}{R T}\right)}{1+(Z-2) \exp \left(-\frac{\varepsilon_{\mathrm{i}}}{R T}\right)}
$$

For convenience we will call the first two terms in eq 1 flexibility terms and all other terms equation-of-state terms. For the evaluation of the equation-of-state terms we will adopt the fluid-lattice approach., ${ }^{4}$ In this approach, each pure $r_{\mathrm{i}}$-mer is characterized by three scaling constants or equation-of-state parameters: A characteristic pressure, $P_{i}^{*}$, a characteristic temperature, $T_{\mathrm{i}}^{*}$, and a characteristic density, $\rho_{\mathrm{i}}^{*}$. An equivalent set of scaling constants is the interaction energy, $\eta_{\mathrm{i}}^{*}$, the number of segments per molecule, $r_{i}$, and the characteristic volume per segment, $v_{i}^{*}$. The basic relation between the scaling constants is

$$
\eta_{\mathrm{i}}^{*}=R T_{\mathrm{i}}^{*}=P_{\mathrm{i}}^{*} v_{\mathrm{i}}^{*}
$$

For our mixture and in the one-fluid approximation the total volume of the system is

$$
V=N_{\mathrm{r}} v^{*}
$$


where $v^{*}$ is the average close-packed volume per segments in the mixture, assumed to be given by the common quadratic mixing rule ${ }^{9}$

$$
v^{*} \varphi_{1}{ }^{2} v_{1}^{*}+2 \varphi_{1} \varphi_{2} v_{12}^{*}+\varphi_{2}{ }^{2} v_{2}^{*}
$$

The segment fraction $\varphi_{\mathrm{i}}$ is given by

$$
\varphi_{\mathrm{i}}=\frac{r_{\mathrm{i}} N_{\mathrm{i}}}{N_{1} r_{1}+N_{2} r_{2}}=\frac{r_{\mathrm{i}} N_{\mathrm{i}}}{r N}=\frac{x_{\mathrm{i}} r_{\mathrm{i}}}{r}
$$

where $x_{\mathrm{i}}$ is the mole fraction of component $\mathrm{i}$. $v_{12}^{*}$ is assumed to be given by

$$
v_{12}^{*}=\left(\frac{v_{1}^{* 1 / 3}+v_{2}^{* 1 / 3}}{2}\right)^{3}
$$

as for hard spheres. Notice that with the mixing rule of eq 7 the close-packed volume is not, generally, conserved during mixing.

The reduced volume is

$$
\tilde{v}=\frac{N_{\mathrm{r}} v^{*}}{r N v^{*}}=\frac{\rho^{*}}{\rho}=\frac{1}{\tilde{\rho}}
$$

where $\rho$ is the density of the mixture.

The intermolecular potential energy of the system is given by ${ }^{9}$

$$
\begin{aligned}
-E_{\mathrm{o}} & =\frac{r N \eta^{*}}{\tilde{v}} \\
& =\frac{r N}{\tilde{v}}\left(\varphi_{1} \eta_{1}{ }^{*}+\varphi_{2} \eta_{2}^{*}-\varphi_{1} \varphi_{2} R T X_{12}\right)
\end{aligned}
$$

where

$$
X_{12}=\frac{\eta_{1}{ }^{*}+\eta_{2}{ }^{*}-2 \xi_{12} \sqrt{\eta_{1}^{*} \eta_{2}^{*}}}{R T}
$$

The binary parameter $\xi_{12}$ is expected to have values close to unity and can be estimated from experimental data for various thermodynamic properties of the mixture.

In terms of the reduced quantities of the mixture the equation of state obtained from eq 1 is $^{9}$

$$
\frac{\tilde{P} \tilde{v}}{T}=\frac{1}{r}-1-\frac{\ln (1-\tilde{\rho})}{\tilde{\rho}}-\frac{\tilde{\rho}}{\tilde{T}}
$$

where

$$
\tilde{P}=\frac{P}{P^{*}} \quad \text { and } \quad \tilde{T}=\frac{T}{T^{*}}
$$

An equation analogous to eq 5 is valid be-

\begin{tabular}{|c|c|c|c|c|c|}
\hline \multirow{2}{*}{ Polymer } & $T^{*}$ & $P^{*}$ & $\rho^{*}$ & $T_{\mathrm{g}}$ & $\varepsilon / R$ \\
\hline & $\mathrm{K}$ & $\mathrm{mN} \mathrm{m}^{-2}$ & $\mathrm{~kg} \mathrm{~m}^{-3}$ & $\mathbf{K}$ & $\mathrm{K}$ \\
\hline Poly(dimethyl siloxane) & 476 & 302 & 1104 & 150 & 177.2 \\
\hline Poly( $\varepsilon$-caprolactone) (PCL) & 570 & 500 & 1189 & 202 & 258.8 \\
\hline Polyisobutylene & 643 & 354 & 974 & 243 & 321.5 \\
\hline Poly(vinyl methyl ether) (PVME) & 639 & 679 & 1115 & 244 & 325.8 \\
\hline Poly(vinyl chloride) (PVC) & 661 & 754 & 1485 & 355 & 597.2 \\
\hline Polystyrene (PS) & 735 & 357 & 1105 & 373 & 602.4 \\
\hline Poly(methyl methacrylate) & 696 & 503 & 1269 & 395 & 689.3 \\
\hline Poly(2,6-dimethylphenylene oxide) (PPO) & 739 & & 1186 & 485 & 936.9 \\
\hline
\end{tabular}
tween $\eta^{*}, P^{*}, v^{*}$, and $T^{*}$ for the mixture.

The total configurational entropy of the system obtained from eq 1 is

$$
\begin{aligned}
\frac{S}{r R N}= & (\tilde{v}-1) \ln \frac{\tilde{v}}{\tilde{v}-1}+\frac{\ln \tilde{v}}{r} \\
& +\frac{\ln Z}{r}+\frac{1}{r} \ln \frac{r}{2}+\frac{1}{r}-1 \\
& -\frac{x_{1} \ln x_{1}+x_{2} \ln x_{2}}{r} \\
& +\frac{x_{1}\left(r_{1}-2\right)}{r}\left[\frac{f_{1} \varepsilon_{1}}{R T}-\ln \left(1-f_{1}\right)\right] \\
& +\frac{x_{2}\left(r_{2}-2\right)}{r}\left[\frac{f_{2} \varepsilon_{2}}{R T}-\ln \left(1-f_{2}\right)\right]
\end{aligned}
$$

In the case of high polymers this equation simplifies to the equation

Table I. Characteristic parameters for pure polymers 


\section{G. Panayiotou}

$$
\begin{aligned}
\frac{S}{r R N}= & (\tilde{v}-1) \ln \frac{\tilde{v}}{\tilde{v}-1} \\
& -1+\varphi_{1}\left[\frac{f_{1} \varepsilon_{1}}{R T}-\ln \left(1-f_{1}\right)\right] \\
& +\varphi_{2}\left[\frac{f_{2} \varepsilon_{2}}{R T}-\ln \left(1-f_{2}\right)\right]
\end{aligned}
$$

The suggestion of Gibbs and DiMarzio is that the entropy $S$ is becoming zero at the glass transition temperature. By adopting this suggestion, one can use eq 15 for the case of pure polymers and evaluate the flex energies $\varepsilon_{\mathrm{i}}$. Values of $\varepsilon_{\mathrm{i}}$ along with equation-of-state parameters for some common polymers are shown in Table I.

It is worth observing in this table, that the quantity $\varepsilon / R T_{\mathrm{g}}$ varies, to a good approximation, linearly with $T_{\mathrm{g}}$. The least squares line is

$$
\frac{\varepsilon}{R T_{\mathrm{g}}}=0.797+2.34 \times 10^{-3} T_{\mathrm{g}}
$$

with a correlation coefficient equal to 0.990 .

If $\varepsilon_{1}, \varepsilon_{2}$, and $\xi_{12}$ are known, eq 15 or $15 \mathrm{a}$ may be used for an estimation of the composition dependence of glass transitions in one phase polymer mixtures. It would be more appropriate to say that, at $T_{\mathrm{g}}, S / r R N=$ $s_{\mathrm{g}} / r$, where $s_{\mathrm{g}}$ is of the order of $-\left(\varphi_{1} \ln \varphi_{1}+\right.$ $\left.\varphi_{2} \ln \varphi_{2}\right)$. In our case $(r \rightarrow \infty)$, on inspection of eq $15, s_{\mathrm{g}} / r$ is essentially zero.

\section{APPLICATIONS}

In this section we will compare theoretical estimations of glass transition temperatures with experimental data for pairs of high polymers known to be compatible in the complete range of compositions. Such a comparison requires the following experimental information:

a) PVT data over extended range of external conditions for each pure polymer in order to evaluate the pure component equation-of-state parameters.

b) Experimental data for any (in principle) basic thermodynamic quantity of mixing of the polymer pair in order to evaluate their binary(ies) parameter(s).

c) Data for the chain flexibility change on mixing the two polymers. In addition one must, of course, have:

d) Experimental data on glass transition temperatures for various compositions of the polymer mixture. Unfortunately the available experimental information for any compatible pair of polymers is, by no means, complete.

The first polymer mixture in which we will apply the above theory is poly(vinyl chloride)-poly(e-caprolactone). This pair of polymers is known to be compatible in the complete range of compositions. ${ }^{17}$ Glass transition temperatures for various compositions have been measured by Koleske and Lundberg. ${ }^{18}$ Estimations of Flory-Huggins $\chi$ interaction parameter at various compositions are also available. ${ }^{19,20}$ Unfortunately, good quality PVT data over extended range of external conditions for both PVC and PCL are not available and their equation-of-state parameters reported in the Table have been estimated from a very limited set of data. ${ }^{19}$ On the basis of the available experimental information $^{19,20}$ the maximum value of the binary interaction parameter $\xi_{12}$ is 1.065 and the minimum value is 0.957 .

In Figure 1 we present various theoretical estimations of glass transition temperatures for this polymer mixture along with the experimental data. ${ }^{18}$ Curves 1,2 , and 3 show the effect of varying $\xi_{12}$ on glass transition temperatures $\left(\xi_{12}\right.$ is equal to $1.0,0.957$, and 1.065 for curves 1,2 , and 3 respectively). Curve 3 indicates that stronger intermolecular interactions (higher values of $\xi_{12}$ ) between the constituents of the mixture lead to maximum deviations of $T_{\mathrm{g}}$-composition data from linearity and this is in accordance with physical intuition. Curve 3 seems to indicate that for an adequate representation of experimental data a lower value of $\xi_{12}$ is needed. However 


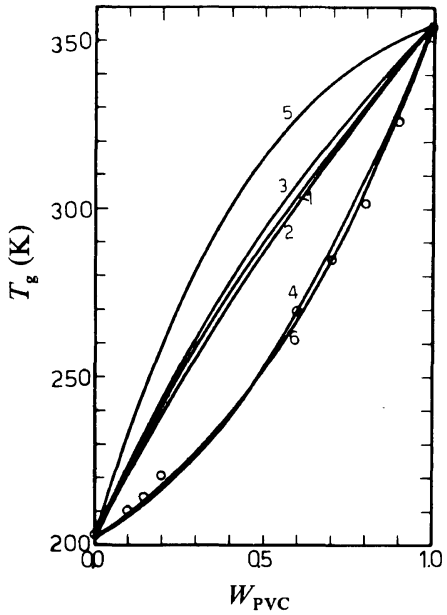

Figure 1. Glass transition temperatures for the mixture PVC-PCL vs. the weight fraction of PVC. $\mathrm{O}$, experimental points. ${ }^{18}$ Curves 1 to 6 are theoretical estimations of $T_{\mathrm{g}}$ for the mixture and have been obtained as described in the text.

lower values of $\xi_{12}$ violate the phase stability (spinodal) condition ${ }^{9}$ for the mixture. Consideration of $v_{12}^{*}$ in eq 9 as an adjustable binary parameter does not improve significantly the model. Curves 1, 2, and 3 were calculated by assuming that the chain flexibilities of the two polymers remained unchanged upon mixing. Relaxation of this later assumption can lead to agreement with experiment. Curve 4 was calculated by assuming a simple linear decrease of the flex energy of the less flexible PVC with composition:

$$
\frac{\varepsilon_{\mathrm{PVC}}}{R}=597.2-325 x_{\mathrm{PCL}}
$$

while $\varepsilon_{\mathrm{PCL}}$ was considered constant. $x_{\mathrm{PCL}}$ is the mole fraction of PCL in the mixture. An increase of the flex energy of the more flexible PCL as

$$
\frac{\varepsilon_{\mathrm{PCL}}}{R}=258.8+325 x_{\mathrm{PVC}}
$$

while keeping $\varepsilon_{\mathrm{PVC}}$ constant gives curve 5 . Both curves 4 and 5 were calculated with $\xi_{12}=1$. A simultaneous consideration of chain flexibility change for both polymers can lead, also, to a better agreement with experiment.

The other possibility is to consider the effect of temperature on chain flexibility. Flex energies may be considered flex free energies in analogy with free energies of interaction ${ }^{8}$ as a consequence of the averaging procedures over microstates involved in formulating the partition function. In other words, we may write

$$
\varepsilon=\varepsilon_{\mathrm{h}}-T_{\varepsilon \mathrm{s}}
$$

Notice that segments of the polymers in the present treatment are fictitious and that "bond" conformations are considered independent of the conformations of previous bonds.

Once a value for $\varepsilon_{\mathrm{s}}\left(\right.$ or $\left.\varepsilon_{\mathrm{h}}\right)$ is assumed, $\varepsilon_{\mathrm{h}}$ (or $\varepsilon_{\mathrm{s}}$ ) is obtained from the $T_{\mathrm{g}}$ of the polymer. Use of eq 18 leaves unaltered eq 4 . However in eq 15 and 15a the flex energies must be replaced by the "enthalpic" parts, $\varepsilon_{\mathrm{h}}$.

In the case of the mixture PVC-PCL and for simplicity, we considered the effect of temperature on the flexibility of the more flexible component, PCL. Curve 6 in Figure 1 was obtained by setting for the flex energy of PCL:

$$
\frac{\varepsilon_{\mathrm{PCL}}}{R}=586-0.962 T
$$

while keeping $\varepsilon_{\mathrm{PVC}}$ constant and $\xi_{12}=1.0$.

The second polymer mixture in which we apply the theory is polystyrene-poly $(2,6-\mathrm{di}$ methylphenylene oxide), also, known to be compatible in the complete range of compositions. ${ }^{17}$ Glass transition temperatures for various compositions have been measured by Kwei and Frish. ${ }^{22}$ PVT data over extended range of external conditions are available for polystyrene. However for PPO the available PVT data are very limited and in fact they allow an estimation of only $T^{*}$ and $\rho^{*}$. Variation of $P^{*}$ from 200 to $500 \mathrm{mN} \mathrm{m}^{-2}$ does not affect significantly the estimated flex energy for PPO. 


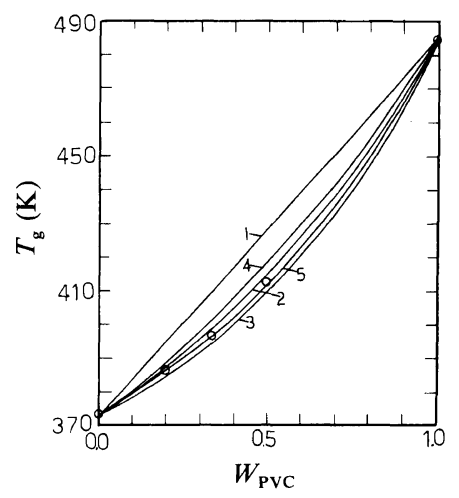

Figure 2. Glass transition temperatures for the mixture PS-PPO vs. the weight fraction of PVC. O, experimental points. ${ }^{22}$ Curves 1 to 5 are theoretical estimations of $T_{\mathrm{g}}$ for the mixture and have been obtained as described in the text.

In Figure 2 we present various theoretical estimations of $T_{\mathrm{g}}$ for this polymer mixture along with the experimental data. ${ }^{21}$ Curve 1 was calculated with $\xi_{12}=1.0$ and $P_{\mathrm{PPO}}^{*}=$ $359 \mathrm{mN} \mathrm{m}^{-2}$ so that $P^{*} / T^{*}$ is the same for both PS and PPO. Agreement with experimental data is reached by a much lower value of $P_{\text {PPO }}^{*}$. Curve 2 is calculated with $\xi_{12}=1.0$ and $P_{\mathrm{PPO}}^{*}=210 \mathrm{~m} \mathrm{~N} \mathrm{~m}^{-2}$. Curves 3 and 4 are, also, calculated with this value of $P_{\mathrm{PPO}}^{*}$ but with $\xi_{12}$ equal to 0.95 and 1.05 respectively. Such a value for $P_{\mathrm{PPO}}^{*}$ is, rather, too low. The characteristic volume per segment for PPO corresponding to this value of $P^{*}$ is $29.3 \mathrm{~cm}^{3}$ $\mathrm{mol}^{-1}$ which is significantly larger than $17.1 \mathrm{~cm}^{3} \mathrm{~mol}^{-1}$ which is the largest reported value of $v^{*}$ for polymers ${ }^{4}$ and still larger than $20.9 \mathrm{~cm}^{3} \mathrm{~mol}^{-1}$ which is the largest reported value of $v^{*}$ for $r$-mers. ${ }^{23}$ Adoption of higher values for $P_{\mathrm{PPO}}^{*}$ necessitate as in the previous case consideration of chain flexibility change upon mixing in order to correlate satisfactorily the experimental $T_{\mathrm{g}}$ 's.

For this mixture we may, also, use eq 18 to describe the flex energy for the more flexible polystyrene. Curve 5 in Figure 2 was obtained by setting

$$
\frac{\varepsilon_{\mathrm{PS}}}{R}=1101-0.842 T
$$

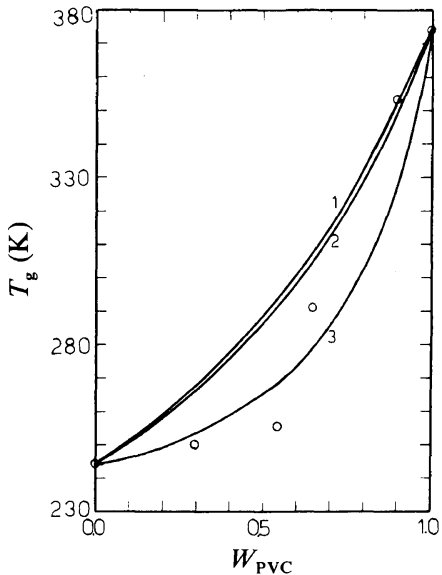

Figure 3. Glass transition temperatures for the mixture PS-PVME $v s$. the weight fraction of PS. O, experimental points. ${ }^{24}$ Curves 1 to 3 are theoretical estimations of $T_{\mathrm{g}}$ for the mixture and have been obtained as described in the text.

while keeping $\varepsilon_{\mathrm{PPO}}$ constant, $\xi_{12}=1.0$ and $P_{\mathrm{PPO}}^{*}=359 \mathrm{~m} \mathrm{~N} \mathrm{~m}^{-2}$.

As a third example we apply the theory to the mixture polystyrene-poly(vinyl methyl ether), also, known to be compatible in the complete range of compositions. ${ }^{17}$ FloryHuggins $\chi$ interaction parameters for this mixture are available. ${ }^{20}$ Glass transition temperatures for various compositions have been measured by Kwei, Nishi, and Roberts. ${ }^{24}$ PVT data for PVME are very limited ${ }^{25}$ and the estimation of the scaling constants of the Table are based on this set.

In Figure 3 we present various theoretical estimations of $T_{\mathrm{g}}$ 's for this polymer mixture along with the experimental data. ${ }^{23}$ Curve 1 was calculated with $\xi_{12}=1.0$ while curve 2 with $\xi_{12}=0.95$. Once again the theory and the experiment cannot be reconciled by considering chain flexibilities unaffected upon mixing. Considerably lower values of $\xi_{12}$ will violate the phase stability condition ${ }^{9}$ for the mixture.

Consideration of the effect of temperature on the chain flexibility of the more flexible PVME leads to curve 3 in Figure 3 which was obtained by setting $\xi_{12}=1.0$ and 


$$
\frac{\varepsilon_{\mathrm{PVME}}}{R}=758.2-1.083 T
$$

while keeping constant $\varepsilon_{\mathrm{PS}}$.

\section{DISCUSSION AND CONCLUSIONS}

In this work we have combined a recent fluid lattice model ${ }^{9}$ of $r$-mer fluid mixtures with the Gibbs-DiMarzio ${ }^{12,13}$ theory of glass transition in order to estimate the composition dependence of $T_{\mathrm{g}}$ 's in one-phase polymer mixtures. The fluid lattice model has allowed a separate estimation of the equation-of-state terms consistent with the thermodynamic behavior of the polymer at higher temperatures. It was, then, possible to estimate the significance of the chain flexibility terms to $T_{\mathrm{g}}$. In addition it was possible to test the validity of the assumption of no change of flex energies upon mixing.

The theory has been applied to three polymer mixtures, known to be compatible in the complete range of composition, for which the information needed for calculations was available. From this comparison it has been shown that the assumption of no change of chain flexibilities upon mixing cannot lead to agreement of theory and experiment without violating the phase stability condition. In fact, theoretical estimations of $T_{\mathrm{g}}$ 's even with the lowest permissible value of the interactional binary parameter $\xi_{12}$ are significantly higher than the experimental $T_{\mathrm{g}}$ 's.

Agreement between experimental $T_{\mathrm{g}}$ 's and theoretical estimations with the present model can be reached by allowing for a chain flexibility change to take place either upon mixing or upon changing the temperature. We have not attempted, in this work, to present and justify and detailed model for this change. However, such a change upon mixing, besides being rather, drastic (see eq 16)), would, also, impose serious questions on the validity of traditional thermodynamic models for polymer mixtures since chemical potential is af- fected by this change. ${ }^{25,26,27}$ This problem can be alleviated by considering a temperature dependence of flex energies for pure polymers. Equation 18 requires, however, further justification and in a more consistent approach it must be applied to both components for all mixtures and for any value of $r$.

The above mentioned problems point to the limitations of, both, the fluid lattice models and the Gibbs-DiMarzio approach to glass transition. Polydispersity has not been taken into account in the present work and the segments of the polymers in the fluid-lattice treatment ${ }^{4,9}$ are fictitious. These approximations may, in part, be responsible for the observed discrepancy between theory and experiment. The otherwise required, change of chain flexibilities may be due to a combined effect of the change in temperature and a change in mixture composition. How much contribute each of these changes to the flex energies? It is hoped that the present work will stimulate, both, theoretical and experimental work towards clarifying this question.

\section{REFERENCES}

1. T. G. Fox, Bull. Am. Phys. Soc., 1, 123 (1956).

2. P. R. Couchman, Macromolecules, 13, 1272 (1980); ibid., 15, 770 (1982).

3. M. Goldstein, Macromolecules, 18, 277 (1985).

4. I. C. Sanchez and R. H. Lacombe, Macromolecules, 11, 1145 (1978).

5. L. A. Kleintjens, Ph. D. Thesis, University of Essex (1979).

6. C. Panayiotou and J. H. Vera, Can. J. Chem. Eng., 59, 501 (1981).

7. M. Okada and T. Nose, Polym. J., 13, 399 (1981).

8. C. Panayiotou and J. H. Vera, Polym. J., 14, 681 (1982).

9. C. Panayiotou, Makromol. Chem., in press.

10. E. A. DiMarzio, J. H. Gibbs, P. D. Fleming, and I. C. Sanchez, Macromolecules, 9, 763 (1976).

11. P. D. Gujati and M. Goldstein, J. Chem. Phys., 74, 2596 (1981).

12. J. H. Gibbs and E. A. DiMarzio, J. Chem. Phys., 28, 373 (1958).

13. J. H. Gibbs, "Modern Aspects of the Vitreous State," J. D. McKenzie, Ed., Butterworths, Washington, D.C., 1960, pp 152-187. 


\section{G. Panayiotou}

14. C. Panayiotou and J. H. Vera, J. Polym. Sci., Polym. Lett. Ed., 22, 601 (1984).

15. P. J. Flory, Proc. R. Soc. (London), Ser. A, 234, 60 (1956).

16. E. A. DiMarzio and J. H. Gibbs, J. Polym. Sci., A-1, 1417 (1963).

17. O. Olabisi, L. M. Robeson, and M. T. Shaw, "Polymer-Polymer Miscibility," Academic Press, New York, N. Y., 1979.

18. J. V. Koleske and R. D. Lundberg, J. Polym. Sci., A2, 7, 795 (1969).

19. O. Olabisi, Macromolecules, 8, 316 (1975).

20. C. Panayiotou and J. H. Vera, Polym. J., 16, 89 27. M. L. Huggins, Ann. N. Y. Acad. Sci., 41, 1 (1942). (1984).
21. B. E. Eichinger and P. J. Flory, Trans. Faraday Soc., 64, 2035 (1968).

22. T: K. Kwei and H. L. Frish, Macromolecules, 11, 1267 (1978).

23. I. C. Sanchez and R. H. Lacombe, J. Phys. Chem., 80, 2352 (1976)

24. T. K. Kwei, T. Nishi, and R. F. Roberts, Macromolecules, 7, 667 (1974).

25. A. Robard, Ph. D. Thesis, McGill University, Canada (1978).

26. C. Panayiotou, J. Chem. Soc., Faraday Trans. 2, 80, 1435 (1984). 